\title{
Bayesian Variable Selection and Data Integration for Biological Regulatory Networks
}

\author{
Supplemental Materials
}

\section{Expression Data Collection and Manipulation}

The expression data used in our Yeast application was collected from the following published papers:

\begin{tabular}{|cl|}
\hline 1 & Casagrande R, et al. (2000). Mol Cell 5 (4):729-35 \\
2 & Chu S, et al. (1998) Science 282(5389):699-705 \\
3 & DeRisi JL, et al. (1997) Science 278(5338):680-6 \\
4 & Diehn M et al.(2000) Nat Genet 25:58-62 \\
5 & Gasch AP, et al. (2000) Mol Biol Cell 11(12):4241-57 \\
6 & Gasch AP, et al. (2001).Mol Biol Cell 12(10):2987-3003 \\
7 & Gross C, et al. (2000) J Biol Chem 275(41):32310-6 \\
8 & Keller G, et al. (2001) J Biol Chem 276(42):38697-702 \\
9 & Kuhn KM, et al. (2001) Mol Cell Biol 21(3):916-27 \\
10 & Ogawa N et al.(2000) Mol Biol Cell 11:4309-21 \\
11 & Protchenko O, et al. (2001) J Biol Chem 276(52):49244-50 \\
12 & Roberts CR, et al. (2000) Science 287:873:80 \\
13 & Rutherford JC, et al. (2001) Proc Natl Acad Sci U S A 98(25):14322-7 \\
14 & Spellman PT et al.(1998) Mol Biol Cell 9:3273-97 \\
15 & Sudarsanam P et al.(2000) Proc Natl Acad Sci U S A 97:3364-9 \\
16 & Yoshimoto H, et al. (2002). J Biol Chem 277(34):31079-31088 \\
17 & Yun CW, et al. (2000) J Biol Chem 275(14):10709-15 \\
18 & Zhu G, et al. (2000) Nature 406(6791):90-4 \\
\hline
\end{tabular}

\section{ChIP Binding Data Collection and Manipulation}

The ChIP binding data was taken from Lee et al. (2002) for our Yeast application. The results produced by a typical ChIP binding experiment for TF $j$ is a set of log-transformed measures $Y_{i}$ for the enrichment of each gene $i$ for that TF $j$. Often these measures are averages calculated over several repetitions of the experiment. These measures are typically standardized, $X_{i}=\left(Y_{i}-\bar{Y}\right) / s_{Y}$, to have a common mean and standard deviation. For each $X_{i}$, a significance test is performed against a null hypothesis of no enrichment, giving a $\mathrm{p}$-value $p_{i}$ for each gene that is calculated using a standard normal distribution or $t$ distribution. For yeast, the ChIP binding data is available in the form of $\mathrm{p}$-values $p_{i}$ calculated using a standard normal distribution. These $\mathrm{p}$-values can not be directly interpreted as the probability $b_{i}=\mathrm{P}(\mathrm{TF} j$ binds gene $i)$ which is needed by our model, 
Figure 1: Mixture model for transcription factor ABF1

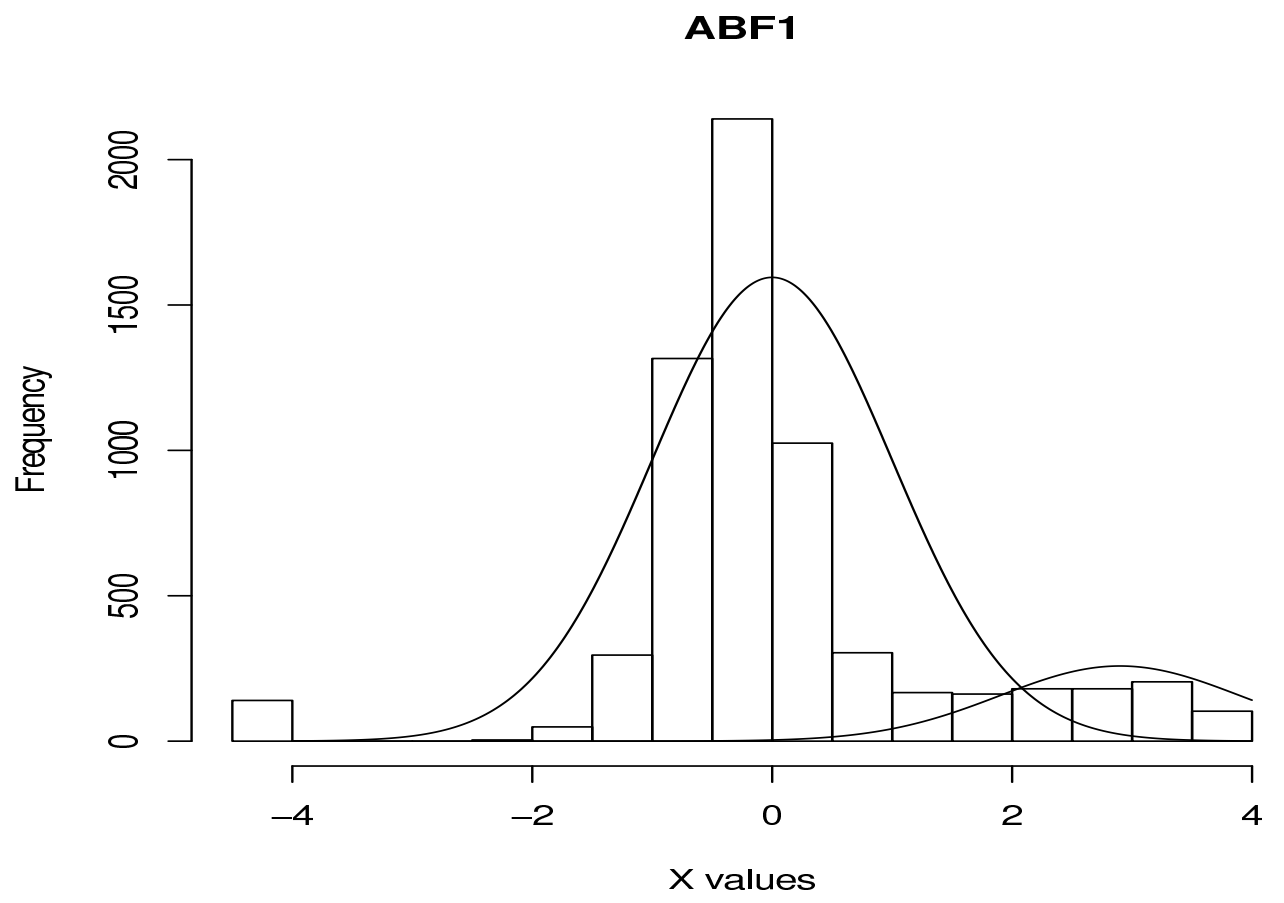

so we use the following procedure to convert $p_{i}$ into binding probabilities $b_{i}$. We first convert the $\mathrm{p}$-values $p_{i}$ back to their corresponding standardized enrichment measures $X_{i}$ using the inverse-CDF for the standard normal distribution. The distribution of these enrichment measures $X_{i}$ should be a mixture of two different groups: a large group of unenriched genes that should be centered at 0 and a smaller group of genes that are truly enriched, with center $\mu>0$ and variance $\tau^{2}$. This mixture model is visually represented for the transcription factor ABF1 in Figure 1. Statistically, we can model each gene with a latent variable $I_{i}$ that indicates whether that gene is in the enriched group $\left(I_{i}=1\right)$ or unenriched group $\left(I_{i}=0\right)$. Our desired binding probabilities for each gene are then simply $b_{i}=\mathrm{P}\left(I_{i}=1\right)$. An EM algorithm (Dempster et al., 1977) was written to simultaneously estimate the unknown parameters of our mixture model (mixing proportion and mean/variance of enriched component) as well as the $b_{i}=\mathrm{P}\left(I_{i}=1\right)$ for each gene. This algorithm alternates between

1. estimating the unknown parameters of our mixture model given current estimates of each $b_{i}$ :

$$
\hat{\mu}=\frac{\sum_{i} I_{i} \cdot X_{i}}{\sum_{i} I_{i}} \quad \hat{\tau}^{2}=\frac{\sum_{i} I_{i} \cdot\left(X_{i}-\mu\right)^{2}}{\sum_{i} I_{i}} \quad \hat{\lambda}=\frac{\sum_{i} I_{i}}{n}
$$


2. estimating the unknown $b_{i}$ 's given current estimates $\hat{\mu}$ and $\hat{\lambda}$ using Bayes rule:

$$
\begin{aligned}
b_{i}=P\left(I_{i}=1 \mid X_{i}\right) & =\frac{P\left(X_{i} \mid I_{i}=1\right) P\left(I_{i}=1\right)}{P\left(X_{i} \mid I_{i}=1\right) P\left(I_{i}=1\right)+P\left(X_{i} \mid I_{i}=0\right) P\left(I_{i}=0\right)} \\
& =\frac{\hat{\tau}^{-1} \exp \left(\frac{1}{2 \hat{\tau}^{2}}\left(X_{i}-\hat{\mu}\right)^{2}\right) \cdot \hat{\lambda}}{\hat{\tau}^{-1} \exp \left(\frac{1}{2 \hat{\tau}^{2}}\left(X_{i}-\hat{\mu}\right)^{2}\right) \cdot \hat{\lambda}+\exp \left(\frac{1}{2} X_{i}^{2}\right) \cdot(1-\hat{\lambda})}
\end{aligned}
$$

until these values converge to a fixed point. This procedure is similar to the non-Bayesian approach of Efron (2004) which also involves the use of a mixture model that is calibrated by the false discovery rate (FDR) instead of being estimated by an EM algorithm. Another detailed discussion of these mixture models is given in McLachlan et al. (2006). It should be noted that our mixture model used the theoretical standard normal null distribution instead of an empirical null distribution since the use of an unrestricted mixture model (with an empirically-fitted null distribution) lead to unreasonable mixtures for several transcription factors. This procedure was repeated for each TF $j$ to generate our full set of binding probabilities $b_{i j}$. The correspondence between the number of genes we predicted as enriched based on p-values $\left(p_{i}<0.005\right)$ and binding probabilities $\left(b_{i}>0.5\right)$ is very good, with a correlation of 0.97 between the number of genes predicted across our 39 transcription factors.

\section{Promoter Element Data Collection and Manipulation}

The first step in calculating a probability $m_{i j}$ for a promoter element occurrence for TF $j$ near to gene $i$ is to scan the genomic sequence nearby to gene $i$ for good matches to the position weight matrix (PWM) for TF $j$. The PWMs for transcription factors for our Yeast application were taken from the Transfac database (Matys et al., 2003). Our scan for good matches is based on a similarity measure between similarity between a TF PWM with width $w$ and a sequence of nucleotides $\left\{a_{1}, \ldots, a_{w}\right\}$ as defined in the equation below:

$$
S\left(a_{1}, \ldots, a_{w}\right)=\sum_{t} \log \left(\frac{P_{t, a_{t}}}{B_{a_{t}}}\right)
$$

where $P_{t, a_{t}}$ is the probability of observing nucleotide $a_{t}$ in position $t$ of the PWM, and $B_{a_{t}}$ is the probability of observing nucleotide $a_{t}$ in the background sequence. When scanning the sequence near gene $i$ for matches to the PWM of TF $j$, we only focus on the score for the best match we find, which we denote as $S_{i j}^{\max }$.

The second step of our procedure is to calculate the probability $m_{i j}$ of a promoter element for TF $j$ near to gene $i$ by comparing the maximum similarity score $S_{i j}^{\max }$ to the scores $S$ that we get by applying our scanning procedure to randomly-generated $w$-mers (sequences of length $w$ ). More specifically, we calculate the probability of $m_{i j}$ of a binding site for TF $j$ near to gene $i$ to be the probability that we observe no score $S$ greater than 
Figure 2: $p^{\prime}$ as a function of $S_{i j}^{\max }$

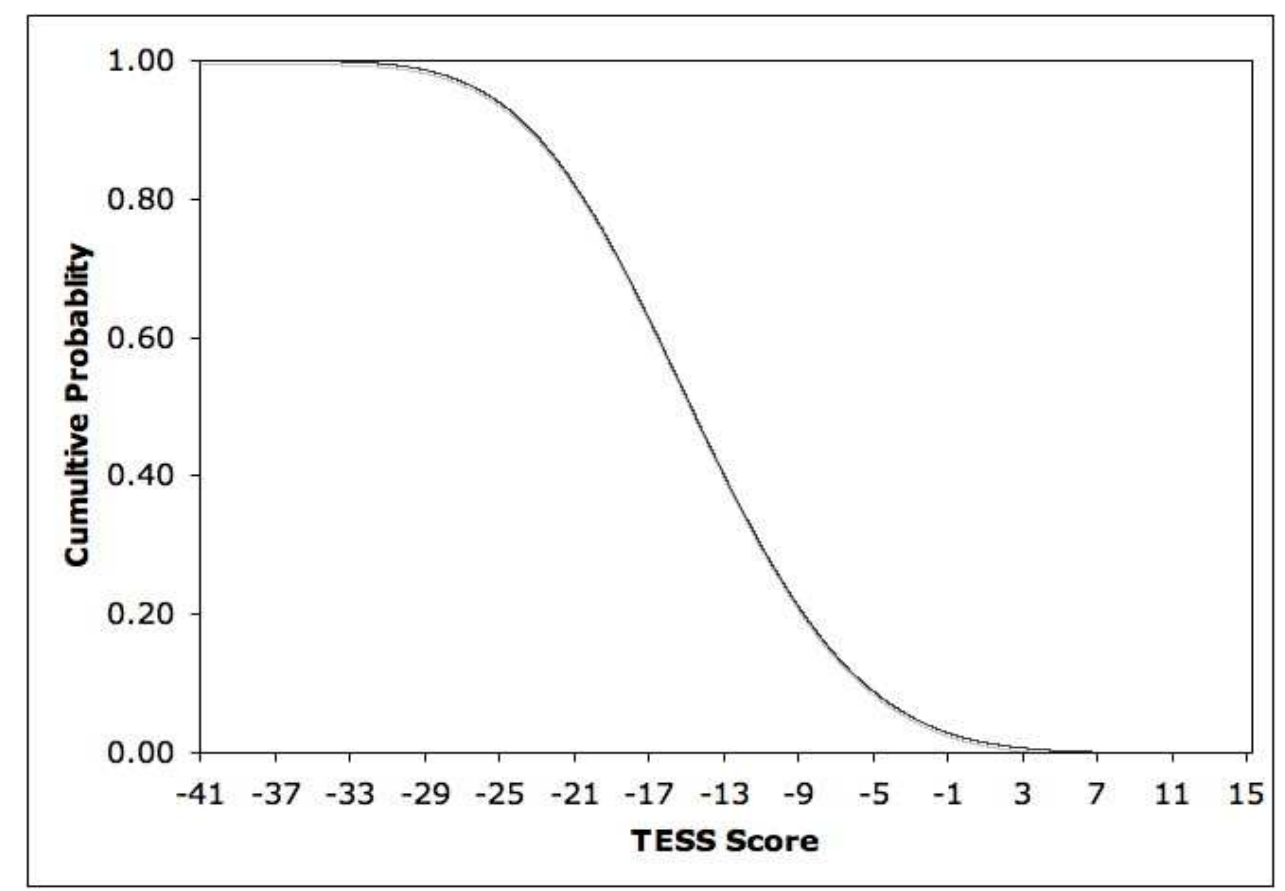

$S_{i j}^{\max }$ in a randomly-generated sequence of length $L$, where $L$ is the length of our original scanned sequence near gene $i$. In other words,

$$
m_{i j}=\left(1-p^{\prime}\right)^{L-w+1}
$$

where $L-w+1$ is the number of possible matches to a PWM of width $w$ in a sequence of length $L$, and $p^{\prime}$ is the probability that a randomly-generated $w$-mer has a score $S$ that is greater than $S_{i j}^{\max }$. As an example, for the C/EBP- $\beta$ PWM, Figure 2 shows the value of $p^{\prime}$ as a function of $S_{i j}^{\max }$. TESS-DIST (Schug and Overton, 1997) was used for the calculation of these binding site probabilities. For short PWMs, this calculation can be done analytically by enumerating all possible $w$-mers and computing their score. However, the number of $w$-mers is $4^{w}$ which becomes cumbersome to enumerate as $w$ increases so TESS-DIST adopts a dynamic programming algorithm to compute the approximate distribution of scores. The approximation is achieved by binning the possible scores thus reducing the total number of possible scores to a number that is linear $(O(w))$ in the length of the PWM.

\section{Evaluating Convergence of Gibbs Sampler}

Multiple chains of our Gibbs sampling algorithm were run from different starting points. Clearly, the large number of parameters in our model rules out an exhaustive visual mon- 
Table 1: $\hat{R}$ values for subsets of expression model parameters.

\begin{tabular}{|cc|cc|}
\hline$\hat{R}\left(\sigma^{2}\right)$ & 1.001 & $\max _{i} \hat{R}\left(\alpha_{i}\right)$ & 1.026 \\
$\max _{j} \hat{R}\left(\beta_{j}\right)$ & 1.014 & $\max _{j} \hat{R}\left(w_{j}\right)$ & 1.009 \\
\hline
\end{tabular}

itoring of convergence for all parameters. Instead, we used the numerical measure $\hat{R}$ (Gelman and Rubin, 1992), which is a measure if the within- vs. between-chain variance that converges towards 1 as the multiple chains converge towards each other. The maximum $\hat{R}$ values (across different parameter subsets) after 5000 iterations are shown in Table 1. Based on a survey of our $\hat{R}$ values, we concluded that our Gibbs sampler chains had converged after 5000 iterations, and our posterior inference is based on 1000 iterations following after these initial 5000 burn-in iterations.

Longer chains were also run and the parameter estimates based on these longer chains showed very little difference when compared to our original results from the shorter chains. In addition to tracking the $\hat{R}$ statistic for our expression model parameters, we also tracked changes in the posterior probabilities $p\left(C_{i j}=1\right)$ for all genes within each chain for the transcription factor HAP4. We found very little change in the posterior probabilities (and hence the inferred target genes) when comparing the samples from our shorter and longer chains, which again indicates that our Gibbs sampler chains have converged by 5000 iterations.

\section{Visual Representation of Regulatory Network}

Figure 3 is a graph of the yeast regulatory network for the 39 transcription factors that were the focus of our analyses. This graph was produced using the aiSee software package $^{1}$. A more detailed version of this same figure is available at:

http://stat.wharton. upenn.edu/ stjensen/research/cogrim.html

\section{Additional Supplementary Files}

We have several additional supplementary files available at:

http://stat.wharton. upenn.edu/ st jensen/research/cogrim.html

\footnotetext{
${ }^{1}$ http: //www.aisee.com/
} 
Figure 3: Regulatory network of inferred target genes for 39 yeast TFs. TFs are indicated by yellow nodes and genes are indicated by blue nodes. Links between gene and TF nodes represent an inferred target gene of a particular TF.

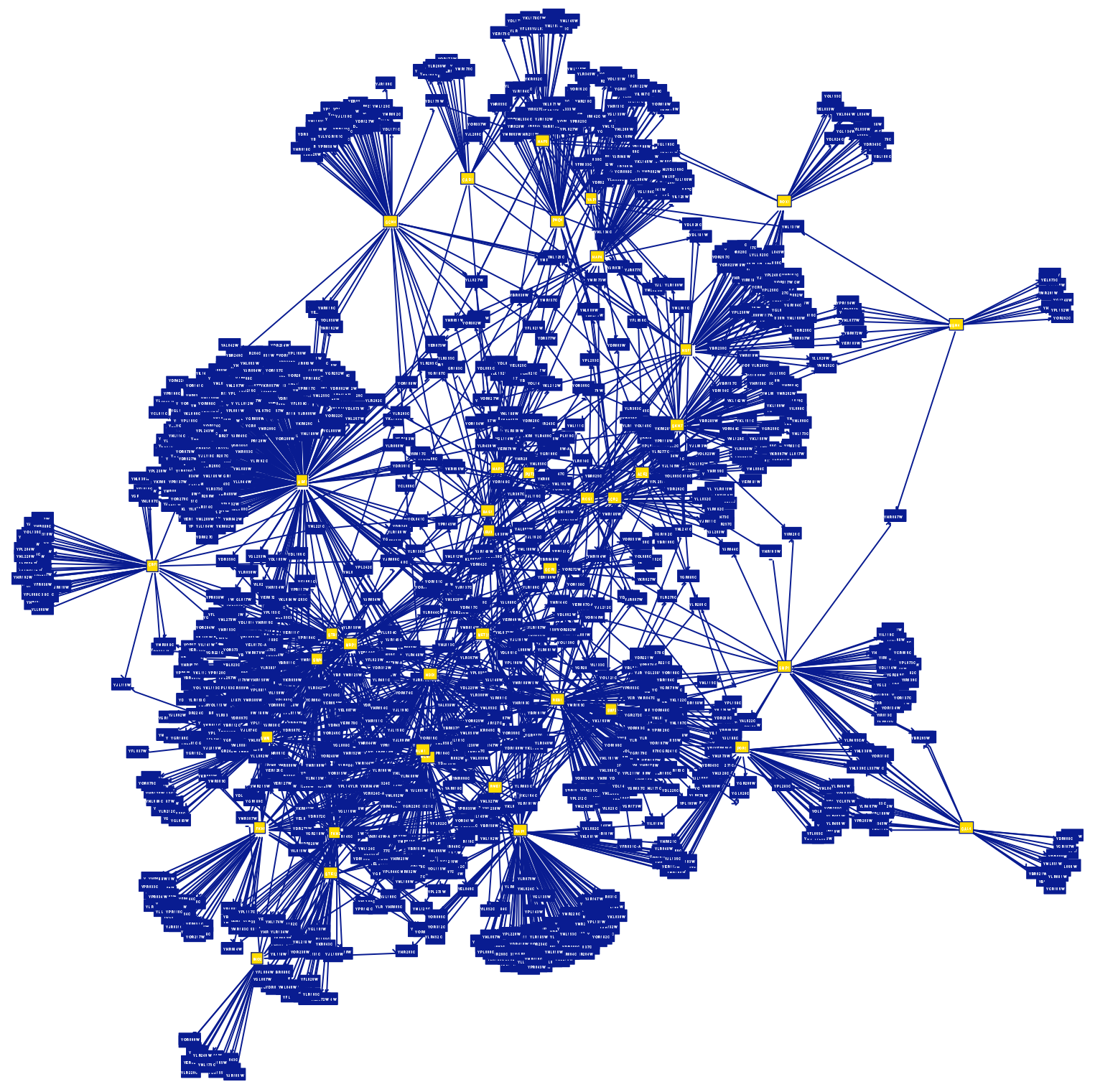


The supplemental file sup. sigbeta.txt contains the seventeen TFs (16 activators and 1 repressor) with significant partial linear effects, where significance for TF $j$ is defined as a $95 \%$ posterior interval for $\beta_{j}$ that does not contain 0 . In addition to the TF names, we also provide information from the SGD database (Hong et al., 2005) that validates our inference that these are true activators or repressors.

The supplemental file sup. siggamma.txt contains the 84 TF pairs with significant interaction effects, where significance for TF pair $j$ and $k$ is defined as a $95 \%$ posterior interval for $\gamma_{j k}$ that does not contain 0 . This file also includes literature references for the subset of inferred interactions that have been already discussed in the previous literature.

The supplemental file sup. validationt fs . txt contains a list of names of the 39 yeast TFs for which external validation information was available that formed the set of yeast TFs examined in detail in our results section.

\section{References}

Dempster, A., Laird, N., and Rubin, D. (1977). Maximum likelihood from incomplete data via the em algorithm. Journal of the Royal Statistical Society, B 39, 1-38.

Efron, B. (2004). Correlation and large-scale simultaneous significance testing.

Gelman, A. and Rubin, D. B. (1992). Inference from iterative simulation using multiple sequences. Statistical Science 7, 457-472.

Hong, E., Balakrishnan, R., Christie, K., Costanzo, M., Dwight, S., Engel, S., Fisk, D., Hirschman, J., Livstone, M., Nash, R., Park, J., Oughtred, R., Skrzypek, M., Starr, B., Theesfeld, C., Andrada, R., Binkley, G., Dong, Q., Lane, C., Hitz, B., Miyasato, S., Schroeder, M., Sethuraman, A., Weng, S., Dolinski, K., Botstein, D., and Cherry, J. (2005). Saccharomyces genome database. Tech. rep., http://www.yeastgenome.org/.

Lee, T., Rinaldi, N., Robert, F., Odom, D., Bar-Joseph, Z., Gerber, G., Hannett, N., Harbison, C., Thompson, C., Simon, I., Zeitlinger, J., Jennings, E., Murray, H., Gordon, D., Ren, B., Wyrick, J., Tagne, J., Volkert, T., Fraenkel, E., Gifford, D., and Young, R. (2002). Transcriptional regulatory networks in saccharomyces cerevisiae. Science 298, 763-764.

Matys, V., Fricke, E., Geffers, R., and et. al. (2003). Transfac: transcriptional regulation, from patterns to profiles. Nucleic Acids Research 31, 374-378.

McLachlan, G., Bean, R., and Jones, L. (2006). A simple implementaton of a normal mixture approach to differential gene expression in multiclass microarrays. Bioinformatics 13, 1608-1615.

Schug, J. and Overton, G. C. (1997). Tess: Transcription element search software on the www. Tech. rep., Technical Report CBIL-TR-1997-1001-v0.0. 